\title{
Solar energetic electron events and coronal shocks
}

\author{
A. Klassen ${ }^{1}$, V. Bothmer ${ }^{2}$, G. Mann ${ }^{1}$, M. J. Reiner ${ }^{3}$, S. Krucker ${ }^{4}$, \\ A. Vourlidas ${ }^{5}$, and H. Kunow ${ }^{2}$ \\ 1 Astrophysikalisches Institut Potsdam, An der Sternwarte 16, 14482 Potsdam, Germany \\ 2 Extraterrestrische Physik, University of Kiel, Kiel, Germany \\ 3 NASA/GSFC, Greenbelt, MD and Center for Solar Physics and Space Weather, The Catholic University \\ of America, Washington DC, USA \\ 4 Space sciences Lab., University of California, Berkeley, Berkeley, CA 94720-7450, USA \\ 5 Naval Research Laboratory, Washington, DC 20375-5320, USA
}

Received 31 July 2001 / Accepted 24 January 2002

\begin{abstract}
Mildly relativistic electrons appear during the solar energetic particle events. A detailed investigation on the origin of such electrons is presented for four particlular solar events. The mildly relativistic electrons have been detected at energies of $0.25-0.7 \mathrm{MeV}$ by COSTEP/SOHO and below $0.392 \mathrm{MeV}$ by Wind 3-DP experiments. Coronal shocks associated with these electron events are identified from the metric-to-decametric solar type II radio bursts. All selected events were associated with solar activity at western longitudes, so that the magnetic footpoints connecting the spacecraft with the Sun were close to the flare/shock/CME site. The associated type II bursts were accompanied by so-called shock accelerated (SA) type III bursts appearing to be emerging from the type II emission site. We found: (1) that all of the $0.25-0.7 \mathrm{MeV}$ electron events were released during or after, but never simultaneously with the onset of type II bursts and CMEs. The time delay between the type II burst onset and the release of the mildly relativistic electrons is in the range of 11.5-45 min; (2) that the mildly relativistic electrons were released rather at the end of SA type III bursts or somewhat later; (3) that the mildly relativistic electrons were released when the associated type II burst and the CME reached a certain height $(h \sim$ 1-4 $R_{\mathrm{s}}$ ) above the photosphere. For the four events studied, it is concluded that mildly relativistic electrons at 0.25- $0.7 \mathrm{MeV}$ energies measured in the interplanetary medium from solar energetic particle events are accelerated by coronal shock waves, commonly in association with white-light CMEs.
\end{abstract}

Key words. Sun: flares - Sun: particle emission - CMEs - Sun: radio radiation - shock waves

\section{Introduction}

Solar energetic electron events observed in interplanetary space are associated with flares and/or coronal mass ejections (CME) (cf. review by Reames 1999). But it is often hard to decide which of these agents is responsible for the generation of mildly relativistic electrons, because both agents can occur independent of each other or together. Furthermore, these agents may be accompanied with coronal and/or interplanetary shock waves.

In the solar corona, shock waves can be generated by two different mechanisms, usually called the piston mechanism and the blast wave mechanism. CMEs can act as a piston, driving bow-shock waves in the corona and into the interplanetary (IP) medium. The blast waves can be produced in the impulsive flare phase due to sudden heating and the pressure pulse (e.g. Vršnak 2001). The type II bursts representing the radio signature of a traveling shock

Send offprint requests to: A. Klassen, e-mail: aklassen@aip.de wave in the corona or IP medium are presumed to be efficient accelerators of suprathermal electrons. So-called herringbone (HB) bursts (e.g. Cairns \& Robinson 1987) and shock accelerated (SA) type III bursts (Cane 1981; Bougeret et al. 1998) show clear evidence of shock accelerated electrons at energies $\leq 30 \mathrm{keV}$. The $\mathrm{HB}$ represent rapidly drifting (type III-like) fine structures emanating from type II lanes towards higher and lower frequencies. The SA type III bursts also start from a type II, but contrary to HB the SA bursts extend into the IP medium and can be measured in situ by spaceborne radio receivers and electron detectors (e.g. Bougeret et al. 1998; Dulk et al. 2000). Both HB and SA bursts are usually interpreted as plasma emission arising from fast electron beams accelerated by shock waves (Roberts 1959).

On the other hand it was argued that shock waves are inefficient accelerators of electrons because the type II bursts have no counterpart in hard X-ray emission (Kahler 1982; Klein et al. 1983). The mildly relativistic electrons 
are better correlated with flare radio (type IV bursts) and hard X-ray emission in the low solar corona than with type II bursts (Klein 1995; Laitinen et al. 2000).

The role of coronal shock waves as accelerators of relativistic electrons is therefore somewhat controversial. On the one hand there is distinct evidence that coronal shocks can produce low energy electrons, on the other hand the relativistic electrons $(\geq 250 \mathrm{keV})$ are not clearly correlated with type II bursts.

The main goal of the present work is to investigate the question: are the coronal shocks able to accelerate mildly relativistic electrons? If so, up to what energies and under which conditions? Therefore we investigated the relative timing of coronal and IP type II bursts, CMEs, SA events and IP electron events for such solar events, at which the manifestation of solar activity in the low corona was relatively weak or absent, so that its contribution to observed relativistic electrons should be considered as being negligible or minimal. But it should be mentioned that the question of the origin (i.e. blast wave or CME driven shock) of the observed shock waves will not be touched in the paper.

In Sect. 2 the criteria of data selection will be described and the instruments providing these data will be briefly introduced. The observational data of the four solar energetic particle events are presented in detail in Sect. 3 . The characteristics of the electron spectra occurring in the solar events studied in this paper are given in Sect. 4 . The results are discussed in Sect. 5 and summarized in Sect. 6 .

\section{Instrumentation and data selection}

The radio ground-based type II observations were obtained with the radio spectrometer of the Potsdam Astrophysical Institut (Mann et al. 1992). The instrument consists of a sweep-frequency spectrometer in the ranges 40-90, 100-170, 200-400 and 400-800 MHz, with a sweep rate of $10 \mathrm{~s}^{-1}$. The spectra are presented after background subtraction. The bright horizontal stripes appearing at a constant frequency are caused by terrestrial disturbances.

The radio space-based observations were obtained with the WAVES instrument on board the Wind spacecraft, which includes several radio receivers that cover the frequency range from $4.09 \mathrm{KHz}$ to $13.825 \mathrm{MHz}$ (Bougeret et al. 1995).

The solar energetic particle events were observed with the Comprehensive Suprathermal and Energetic Particle Analyser (COSTEP) (Müller-Mellin et al. 1995) aboard the Solar and Heliospheric Observatory $(\mathrm{SOHO})$ and with the 3-D plasma instrument on board the Wind spacecraft (Lin et al. 1995). In this study we use the COSTEP and Wind $3-\mathrm{DP}$ electron flux measurements in the ranges 0.25-0.7 MeV and below $0.39 \mathrm{MeV}$, respectively.

White-light observations of the corona were provided by the Large Angle Spectroscopic Coronagraph (LASCO/SOHO) (Brueckner et al. 1995). LASCO consists of three coronographs $\mathrm{C} 1, \mathrm{C} 2$, C3 which image the corona of the Sun from 1.1 to 30 solar radii. Additionally we use images from the Extreme Ultraviolet Imaging Teleskope (EIT) aboard $S O H O$ ) and from the soft X-ray Teleskop (SXT) on bord Yohkoh spacecraft. The soft and hard X-ray flux recordings have been obtained by GOES, HXT/Yohkoh and BATSE/CGRO instruments.

\subsection{Data selection}

The events used in this study have been selected according to the following criteria:

1. all events were associated with solar activity at western longitudes, so that the magnetic footpoints connecting the $\mathrm{SOHO} /$ Wind spacecraft with the Sun were close to the shock/flare/CME site. Therefore the coronal propagation of electrons can be neglected;

2. all associated type II bursts were accompanied by SA type III bursts, emanating from the type II emission;

3. no other type III bursts before, during and after these type II bursts were observed;

4. other radiative signatures of nonthermal energy release in the low corona, such as hard $\mathrm{X}$-ray bursts, radio continua (e.g. type IV emission) or noise storm enhancements were absent or very weak, especially during the relativistic electron injection time.

The criteria (3) and (4) were imposed to eliminate cases in which the high energy electrons might be accelerated at the low corona. Nevertheless for each event the possibility of such an eventual contribution will be discussed.

\section{Observations}

\subsection{Determination of the solar release time (SRT)}

For the determination of the release time of the electrons at the Sun we use the particle data measured by COSTEP and WIND 3-DP. The Solar Release Time (SRT) of electrons $t_{\mathrm{SRT}}$ at the Sun is estimated from the observed rise time (UT) of the electron flux at about $1 \mathrm{AU}\left(t_{\mathrm{AU}}\right)$ as:

$t_{\mathrm{SRT}}=t_{\mathrm{AU}}-L / v$,

where $L$ is the length of the path along the magnetic field spiral from the release site on the Sun up to the spacecraft (i.e. $\approx 1 \mathrm{AU}), v$ denotes the particle velocity. The interplanetary magnetic field (IMF) spiral can be approximated by a Parker spiral whose length depends only on the solar wind speed. We also assume that the particles would propagate scatterfree along the magnetic field spiral with a pitch angle $0^{\circ}$. (Note that the traveling time of an electron would be larger if the pitch angle is greater than $0^{\circ}$.)

The observed time of type II bursts, CMEs and other electromagnetic emission were also expressed in terms of the solar release time according to:

$t_{\mathrm{SRT}}=t_{\mathrm{AU}}-500 \mathrm{~s}$,

$500 \mathrm{~s}$ is the time required for electromagnetic emission to travel from the Sun to the Earth. Note that such transformation is correct only for emission close to the Sun. 
Table 1. Summary of particles, radio, optic, and X-ray observations.

\begin{tabular}{|c|c|c|c|c|c|c|c|}
\hline Observation & $\begin{array}{l}\text { Start, } \\
\text { SRT }\end{array}$ & $\begin{array}{l}\text { Peak, } \\
\text { SRT }\end{array}$ & $\begin{array}{l}\text { End, } \\
\text { SRT }\end{array}$ & Type & $\begin{array}{c}\text { Range, MHz/ } \\
\text { Location (AR) }\end{array}$ & $\begin{array}{l}\text { Speed, } \\
\mathrm{km} \mathrm{s}^{-1}\end{array}$ & Remarks \\
\hline \multicolumn{8}{|c|}{20 April 1998} \\
\hline $\begin{array}{l}\text { COSTEP } \\
\text { Wind 3-DP } \\
\text { Type II } \\
\text {-SA } \\
\text { SXR (goes) } \\
\text { HXR (yohkoh, batse) } \\
\text { CME (lasco) }\end{array}$ & \begin{tabular}{|l|}
$10: 20$ \\
$10: 19 \pm 2$ \\
$09: 48.5$ \\
$09: 52$ \\
$09: 12$ \\
$09: 42.1$ \\
$09: 57^{*}$ \\
\end{tabular} & $\begin{array}{l}\text { 10:13 } \\
09: 42.2\end{array}$ & $\begin{array}{l}10: 52 \mathrm{D} \\
10: 29 \\
11: 10 \\
09: 42.3\end{array}$ & $\begin{array}{c}\text { III } \\
\text { M1.4/- } \\
\text { partial halo }\end{array}$ & $\begin{array}{c}55-4 \mathrm{X} \\
10 \mathrm{U}-1 \mathrm{X} \\
\\
\text { SW-limb }\end{array}$ & 1150 & $\begin{array}{l}V_{\mathrm{sw}}=350 \mathrm{~km} \mathrm{~s}^{-1} \\
B_{\mathrm{o}}=-5.2 \\
\Delta t_{\mathrm{srt}}^{\mathrm{II}-0.7}=30 \mathrm{~min} \\
\text { no bursts at 10:03-10:42 }\end{array}$ \\
\hline \multicolumn{8}{|c|}{19 May 1998} \\
\hline $\begin{array}{l}\text { COSTEP } \\
\text { Wind 3-DP } \\
\text { Type II } \\
\text { - SA } \\
\text { SXR (goes) } \\
\text { HXR (yohkoh) } \\
\text { EIT } \\
\text { CME (lasco) }\end{array}$ & \begin{tabular}{|l|}
$09: 59$ \\
$09: 59 \pm 2$ \\
$09: 43$ \\
$09: 43$ \\
$09: 56$ \\
no burst \\
$<09: 46$ \\
$10: 19^{*}$ \\
\end{tabular} & $10: 16$ & $\begin{array}{l}\text { 10:42D } \\
10: 20 \\
10: 10 \mathrm{D} \\
>10: 10\end{array}$ & $\begin{array}{c}\text { HARM } \\
\text { III } \\
\text { B7.9/- } \\
\text { partial halo }\end{array}$ & $\begin{array}{c}86-6 \\
50-1 \mathrm{X} \\
\\
\\
\mathrm{NW}\end{array}$ & $\begin{array}{l}600 \\
650\end{array}$ & $\begin{array}{l}V_{\mathrm{sw}}=400 \mathrm{~km} \mathrm{~s}^{-1} \\
B_{\mathrm{o}}=-2.2 \\
\Delta t_{\mathrm{srt}}^{\mathrm{II}-0.7}=16.5 \mathrm{~min} \\
\text { no obs. at } 09: 30-09: 56\end{array}$ \\
\hline \multicolumn{8}{|c|}{16 June 1998} \\
\hline $\begin{array}{l}\text { COSTEP } \\
\text { Wind 3-DP } \\
\text { Type II } \\
\text {-SA } \\
\text { SXR (goes) } \\
\text { HXR (batse) } \\
\text { CME (lasco) }\end{array}$ & \begin{tabular}{|l|}
$18: 47$ \\
$18: 55 \pm 6$ \\
$18: 02 \mathrm{U}$ \\
$18: 02$ \\
$17: 55$ \\
$18: 01.3 \mathrm{E}$ \\
$18: 19^{*}$ \\
\end{tabular} & $\begin{array}{l}18: 34 \\
18: 01.5\end{array}$ & $\begin{array}{l}19: 30 \mathrm{D} \\
18: 36 \\
19: 20 \\
18: 06\end{array}$ & $\begin{array}{c}\text { HARM } \\
\text { III } \\
\text { M1.0/- } \\
\text { partial halo }\end{array}$ & $\begin{array}{c}170 \mathrm{U}-.05 \\
70-1 \mathrm{X} \\
\\
\mathrm{SW}\end{array}$ & $330-530$ & $\begin{array}{l}V_{\mathrm{sw}}=400 \mathrm{~km} \mathrm{~s}^{-1} \\
B_{\mathrm{o}}=+1.1 \\
\Delta t_{\mathrm{srt}}^{\mathrm{II}-0.7}=45 \mathrm{~min} \\
\text { no obs. after } 18: 40\end{array}$ \\
\hline \multicolumn{8}{|c|}{18 Februar 2000} \\
\hline $\begin{array}{l}\text { COSTEP } \\
\text { Wind 3-DP } \\
\text { Type II } \\
\text {-SA } \\
\text { SXR (goes) } \\
\text { HXR (yohkoh, batse) } \\
\text { CME (lasco) }\end{array}$ & \begin{tabular}{|l|}
$09: 21$ \\
$09: 22 \pm 2$ \\
$09: 10 \mathrm{E}$ \\
$09: 08.3$ \\
$09: 13$ \\
no bursts \\
$09: 46^{*}$ \\
\end{tabular} & $09: 19$ & $\begin{array}{l}09: 42 \\
09: 25 \\
09: 30\end{array}$ & $\begin{array}{c}\text { HARM } \\
\text { III } \\
\text { C1.1/SF } \\
\text { partial halo }\end{array}$ & $\begin{array}{c}170 \mathrm{U}-40 \mathrm{X} \\
45-1 \mathrm{X} \\
\mathrm{S} 16 \mathrm{~W} 78(8867) \\
\mathrm{W}+\end{array}$ & $580-1020$ & $\begin{array}{l}V_{\mathrm{sw}}=400 \mathrm{~km} \mathrm{~s}^{-1} \\
B_{\mathrm{o}}=-7.0 \\
\Delta t_{\mathrm{srt}}^{\mathrm{II}-0.7}=11.5 \mathrm{~min} \\
\text { no obs. after 09:20 }\end{array}$ \\
\hline
\end{tabular}

SA - shock (type II) accelerated type III bursts, Harm - both harmonics observed, E - event began earlier, D - ended later, $\mathrm{X}$ - extend beyond the instrument range, $\mathrm{U}$ - uncertain frequency/time, $*$ - time corresponding to the first appearance in $\mathrm{C} 2$ coronograph, No obs. - no observation, type II speed is calculated using the 2x Newkirk model, $V_{\text {sw }}$ - solar wind speed using for calculation of the Parker spiral length, $B_{\mathrm{o}}-$ the heliographic latitude of the Earth, $\Delta t_{\mathrm{srt}}^{\mathrm{II}-0.7}$ - time delay between the onset of radio type II burst and the onset of $0.7 \mathrm{MeV}$ electron event.

Since we don't know the direction of propagation of the interplanetary type II and SA type III bursts, the precise distance between the radio sources and the $\mathrm{SOHO} /$ Wind spacecraft is unknown. The approximated time delay between the light propagation time along the Sun-Earth line and the $0.25-0.7 \mathrm{MeV}$ electrons travel time along the Parker spiral is of about $3-6 \mathrm{~min}$.

\subsection{Presentation and scales}

The dynamic radio spectra (e.g. Fig. 2) are presented in $\log$ frequency scale on the left-hand ordinate. On the righthand ordinate the corresponding radial distance from the solar photosphere in solar radii units $\left(R_{\mathrm{s}}=696000 \mathrm{~km}\right)$ is given. The radial distance is calculated assuming radio emission at the fundamental plasma frequency, $f_{\mathrm{p}}(\mathrm{Hz}) \simeq$ $9 \times 10^{3} \cdot n_{\mathrm{e}}^{1 / 2}\left(\mathrm{~cm}^{-3}\right)$. We use the twofold Newkirk (1961) density model up to a distance of $1 R_{\mathrm{s}}$ and the twofold
Mann et al. (1999) model for radial distances of 1-10 $R_{\mathrm{s}}$ above the photosphere of the Sun. The density models deduced for quiet coronal densities near sunspot minimum are multiplied by factor of 2 to simulate an enhanced density region, such as a coronal streamer or an active region. Indeed, it was found in previous observations of type II source heights that the densities agrees roughly with the twofold Newkirk model below 1 solar radii (Steward 1976). Furthermore, the observed start height $\left(0.40-0.54 R_{\mathrm{s}}\right)$ of the type II radio source on 18 February 2000 obtained with Nançay radioheliograph (courtesy NRH team) confirms that the twofold Newkirk model is a relatively good approximation at least for this event. Nevertheless, because the exact density distribution above the other given active regions is unknown and radio imaging observations are not available, the heights of radio sources should only be considered as approximated values. The uncertainities in derived heights and speeds vary in the range of $\mp 35 \%$ 
relatively to the twofold Newkirk model, assuming that the density distribution varies in wide range from onefold to fourfold Newkirk model.

It is important to keep this in mind when comparing the heights of type II sources with the CME positions. The CME position is measured as the distance between its leading edge and the solar photosphere in projection on the plane of sky. In the following, all the data are plotted in terms of the Solar Release Time (SRT).

\subsection{Description of the data in Table 1}

The main parameters of all events are given in Table 1 . In Col. 1, we have listed the observations of high energy electrons in the range $0.25-0.7 \mathrm{MeV}$ (COSTEP) and 0.050$0.392 \mathrm{MeV}$ (Wind 3-DP), radio observations of type II burst (Type II) and shock associated type III bursts (SA), soft (SXR) and hard (HXR) X-ray observations by GOES, HXT/Yohkoh and BATSE/CGRO, and white light CME observations by LASCO/SOHO. The times of start, peak and end for each event in units of SRT are given in Cols. 2-4. The type of coronal type II burst, the SA event, the flare and the CME is given in Col. 5. In Col. 6, we have listed the frequency range of the type II and the SA event, and the position of the flare and the associated CME. The speeds of type II burst and the CME is given in Col. 7. In the remarks we have presented the solar wind speed $V_{\mathrm{sw}}$ using for calculation of the Parker spiral length, $B_{\mathrm{o}}-$ heliographic latitude of the Earth, and $\Delta t_{\mathrm{srt}}^{\mathrm{II}-0.7}$ - time delay between the onset of radio type II burst and the onset of $0.7 \mathrm{MeV}$ electron event.

\subsection{Event on 19 May 1998}

We begin our detailed analysis with the event on 19 May 1998, the simplest one with a good data coverage by all instruments.

The particle event was associated with a filament eruption at co-ordinates N29W46, a metric-decametric type II burst, an EIT wave and a CME (Gopalswamy et al. 2000). After a sudden heating of the filament (in the time interval 09:36.3-09:47 SRT), which is clearly seen on SXT and EIT images (Fig. 1), a weak B7.9 GOES flare occurred at 09:56 and peaked at 10:07 SRT. The X-ray flare was partly associated with the emission from the filament itself and partly from an active region (AR) at the SW limb. There were no flares in $\mathrm{H}_{\alpha}$ and $\mathrm{X}$-rays or radio bursts before the filament eruption. The erupted filament was a quiescent filament and was located between two ARs as seen in $\mathrm{H}_{\alpha}$ images (Fig. 1). Unfortunately, we do not know the exact start time of the filament eruption, due to a data gap of $\mathrm{H}_{\alpha}$ and $\mathrm{X}$-rays observations. But from the available images presented in Fig. 1, it is presumed that the eruption starts between 09:36.3-09:44 SRT. At 09:36.3 SRT the filament is not yet seen in soft $\mathrm{X}$-rays, but at 09:47 SRT the event is already in progress (cf. SXT and EIT data). The fact that the type II burst occurred at 09:44 SRT indicates that the eruption must have started before the type II, because the initiated wave needs sufficient time to reach the height, where the type II burst was generated. We assume that the type II burst (shock wave) was directly caused by a filament heating and eruption that occur between 09:36.3 and 09:44 SRT.

\subsubsection{Radio observations}

The metric-decametric type II burst (visible in both fundamental $(\mathrm{F})$ and harmonic $(\mathrm{H})$ lanes) appeared at 09:43 SRT at 35-60 MHz (F) and ended at 10:42 SRT at $6 \mathrm{MHz}(\mathrm{F})$. It has a multilane structure and consist of two components, i.e. "backbone" and "herringbones" (Fig. 2).

From the type II onset until 10:02 SRT intense shock accelerated (SA) type III bursts emanate from the type II backbone component, drifting towards lower frequencies. Both "herringbones" and SA bursts are fast drifting emissions, the difference between both phenomena is that the "herringbones" do not escape into the interplanetary medium, so that SA bursts are not the extension of the "herringbones".

Furthermore in the kilometric domain the single type III bursts merge together and are visible down to about $20 \mathrm{kHz}$, i.e. close to the local plasma frequency near to Wind spacecraft (1 AU) (not shown in Fig. 2).

Because there are no metric type III radio bursts before and during the entire duration of the type II emission, we suppose that these SA type III bursts were accelerated by the shock wave associated with the type II burst and that they start from the type II radio source.

\subsubsection{Type II height and speed estimation}

From the type II start frequency $f_{\text {st }}=35-60 \mathrm{MHz}$ and drift rate we estimated the start height $\left(0.90-0.58 R_{\mathrm{S}}\right.$ or $\sim 630000-407000 \mathrm{~km})$ and the speed $\left(830 \mathrm{~km} \mathrm{~s}^{-1}\right)$ of the type II using the twofold Newkirk density model. The disturbance should have started earlier than the type II onset at 09:44 SRT, because it needs some time to reach the estimated height where it became a shock wave. The disturbance started at approximately 09:33-09:37 SRT, i.e. 7-11 min before the type II onset. Since we do not know the real density distribution above the filament and the precise position of the type II burst is also unknown (no imaging radio observations are available), the above estimations are only rough values (cf. Sect. 3.2).

Note that all parameters of the type II burst were measured from the harmonic lane and were subsequently normalized to the fundamental one because the latter is not completely seen in the radio spectrometer range.

\subsubsection{Yohkoh/GOES X-ray observations}

In Fig. 1, the second panel shows the soft X-ray evolution before and during the event. The first two images 


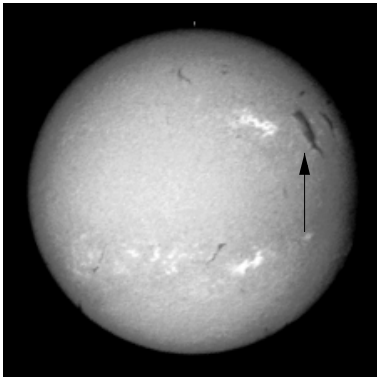

Before: Ha at 09:05

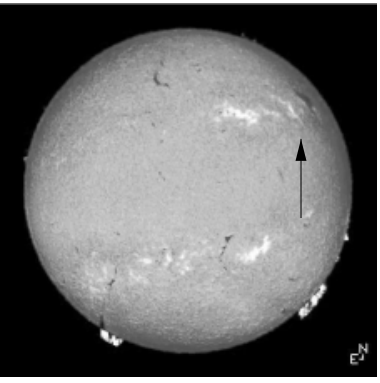

After: Ha at 11:51 SRT

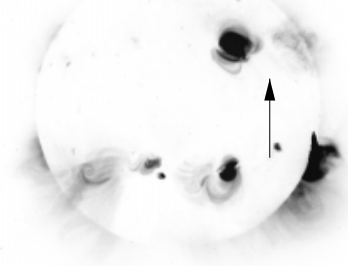

SXT-Yohkoh: 09:21.2

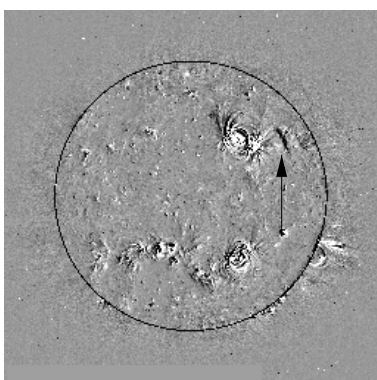

EIT: 09:11-08:54

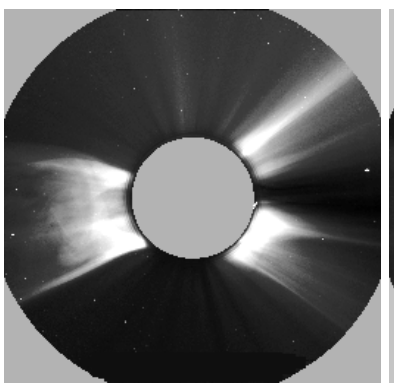

LASCO-C2: 09:20
09:36.3

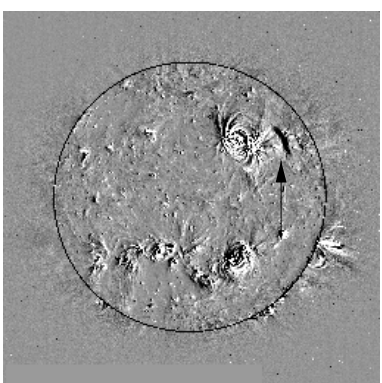

09:25-08:54

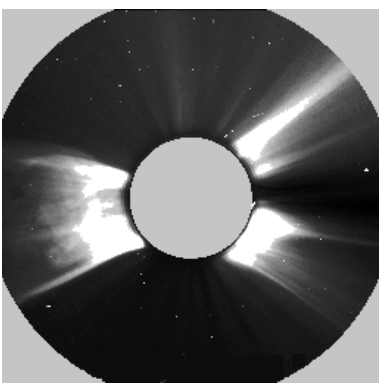

09:54

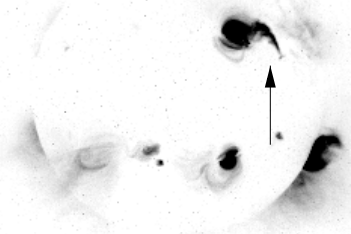

09:47

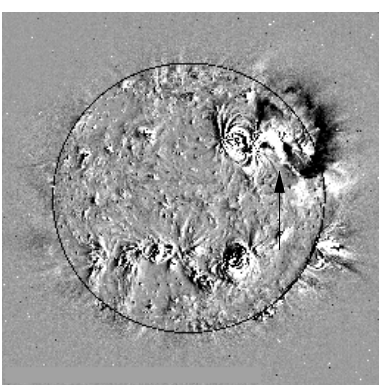

09:47-08:54

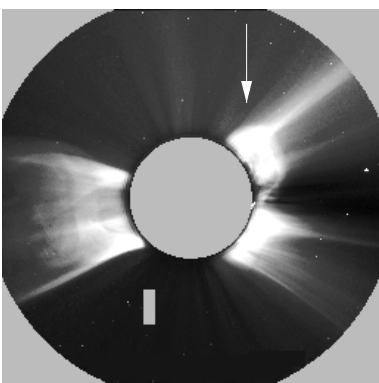

10:19

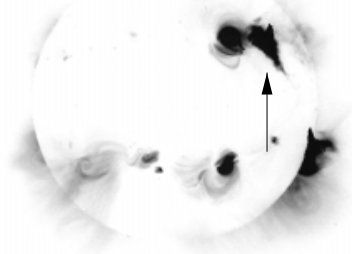

10:20 SRT

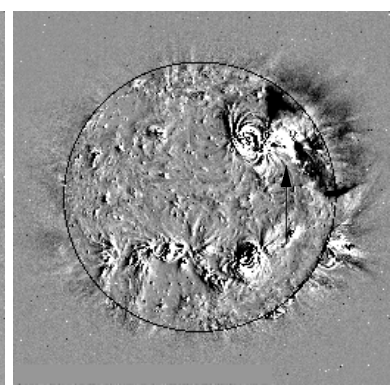

09:59-08:54 SRT

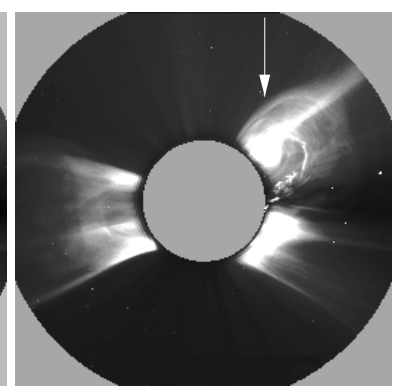

10:54 SRT

Fig. 1. Observations of the filament eruption and the CME on 19 May 1998. Arrows point to the filament position (panels 13). The time is given in Solar Release Time (SRT). Top panel: $\mathrm{H}_{\alpha}$ images (courtesy Learmonth Solar Observatory and Kiepenheuer-Institut für Sonnenphysik) shortly before and after the filament eruption. At 09:05 SRT the filament is seen as a dark feature elongated in NS direction. After the eruption only a weak feature in emission is observed. Second panel: Yohkoh SXT images. Dark means enhanced emission. At 09:21.2-09:36.3 SRT the filament is not yet seen. At 09:47 and 10:20 it was heated and an arcade below and along the filament was formed. Third panel: EIT $195 \AA$ Aifference images (courtesy B. Thompson) showing the filament (first two) and the manifestation of an EIT wave after the filament eruption. Bright means enhanced emission. Bottom panel: CME observed by the LASCO C2 coronograph. Arrows point to the leading edge of the CME. The erupted filament (CME core) occurs inside the CME as a long narrow structure. North is to the top and east to the left.

show no changes in the region close to the filament, but at 09:47 SRT an arcade of soft X-ray loops below and along the filament has already been formed. The heating process starts between 09:36.3 and 09:47 SRT involving arcade formation and filament heating.
In Fig. 2 (bottom panel) the soft $\mathrm{X}$-ray flux (GOES, 1-8 $\AA$ ), its time derivative (dotted), and the corresponding hard X-ray (HXT/Yohkoh) flux are presented. The X-ray GOES flare (start at 09:56, peak at 10:07) appears after the filament eruption and the type II onset. It was 


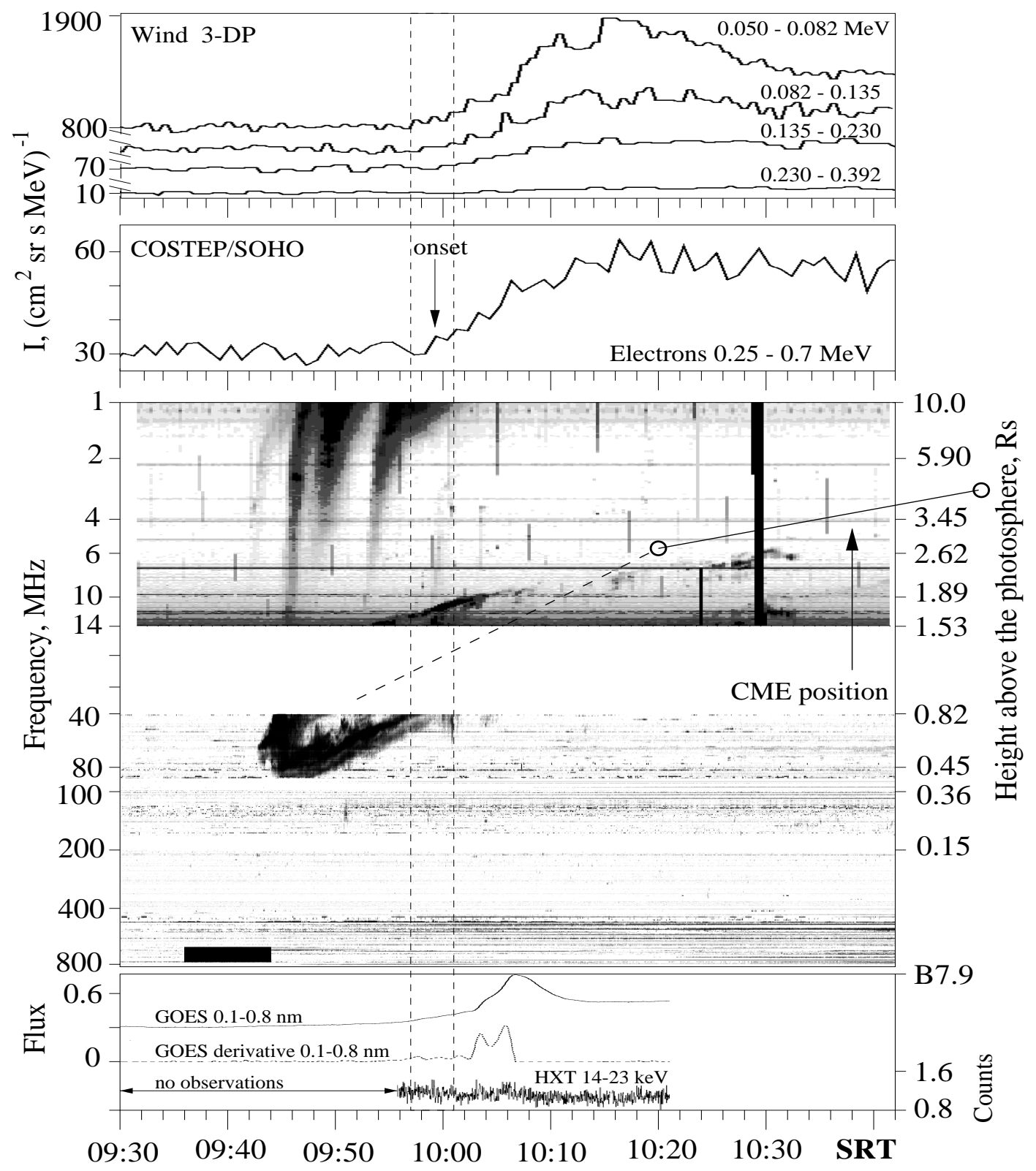

Fig. 2. Event on 19 May 1998. Relation between electron intensities in the range 0.050-0.7 MeV and electromagnetic emission at/close to the Sun. The coronal type II burst, the CME and the electron event start after the filament eruption, see Fig. 1. The energetic electrons in all channels were released about simultaneously, 16.5 min after the type II and the SA type III bursts onset. Top two panels: electron intensities observed by Wind 3-DP and COSTEP instruments. The vertical dashed lines show the onset time interval of electrons detected in the range $0.050-0.392 \mathrm{MeV}$. The arrow indicates the onset time of electrons in the range $0.25-0.7 \mathrm{MeV}$. Middle panel: dynamic radio spectrum (800-1 MHz) overlaid with CME trajectory. A type II burst occur between $86-6 \mathrm{MHz}$ at 09:43-10:42 SRT. From the type II onset, the intense SA type III bursts escape from it lanes. At frequencies above those of type II no other type III, IV were observed. Open circles represent the CME heights (right scale), dashed line is the backwards extrapolation of the CME trajectory. Black bar denotes the time interval of the filament eruption. Bottom panel: soft X-ray flux (GOES: 1-8 $\AA, \mathrm{W} \mathrm{m}^{-2} 10^{6}$ ), its time derivative (dotted, Flux · 50) and hard X-rays (HXT/Yohkoh). The soft X-ray flare starts after the type II onset. The hard X-rays show in agreement with the temporal behavior of the soft X-rays derivative a weak enhancement at 10:03-10:07 SRT after the onset of the electron event.

associated partly with arcade formation/filament brightening, and partly with the emission from an AR on the southwest limb. The hard X-ray (14-23 keV) curve shows in agreement with the temporal behaviour of the soft $\mathrm{X}$ ray derivative a very weak enhancement at 10:03-10:07 SRT after the onset of electron event.

\subsubsection{EIT observations}

In Fig. 1, the third panel presents four difference images obtained between 08:54 and 09:59 SRT. The filament is seen as dark feature (arrow) and becomes darker at 09:11 and 09:25 SRT relative to the reference image on 
08:54 SRT. Such darkening is well known from $\mathrm{H}_{\alpha}$ observations and occurs normally before a filament eruption (Smith \& Ramsey 1964; Martin \& Ramsey 1972). Simultaneously with darkening some material upflow from the filament at 09:11 and 09:25 SRT is observed. On the next image on 09:47 SRT, the EIT wave is already in progress. Some EUV loops occur below and along the filament. Its northern part is in emission (Fig. 1). Thus, the event starts between 09:25 and 09:47 SRT as seen from EIT observations. Suppose that the EIT wave starts between 09:36.3 SRT and the type II starts on 09:44 SRT. Then, the speed of the EIT wave must be $600 \mathrm{~km} \mathrm{~s}^{-1}$ or higher.

\subsubsection{LASCO observations}

The coronal mass ejection (CME) was first detected by the LASCO C2 instrument at 10:19 SRT above the northwest limb (cf. Fig. 1, bottom panel). Its height-time plot is shown in Fig. 2. The CME had a speed of $650 \mathrm{~km} \mathrm{~s}^{-1}$ and the filament had a speed of $457 \mathrm{~km} \mathrm{~s}^{-1}$ in projection of the sky plane. We estimated a start time in the interval 09:34-09:42 SRT by backwards extrapolation of the leading edge trajectories of the CME and the filament.

\subsubsection{COSTEP and Wind 3-DP particle measurements}

The energetic particles were detected by the COSTEP and Wind 3-DP particle instruments in the energy ranges 0.25-0.7 and below $0.39 \mathrm{MeV}$, respectively. The upper panels in Fig. 2 show the corresponding electron intensities. The first electrons were ejected near the Sun at 09:59 SRT. The maximum occurs at 10:16 SRT (COSTEP). From the Wind 3-DP intensity curve (see Krucker et al. 1999), we estimate that the first electrons in all four energy ranges were released at 09:59 $\pm 3 \mathrm{~min}$ SRT. Thus, the two separate estimations of the release time are in agreement. The electrons in the whole energy range were simultaneously released 16.5 min after the onset of the type II and, especially, at the end of the SA type III bursts. Therefore, we suggest that the detected electrons were not related to the electrons producing the SA type III bursts. The same result was found by Krucker et al. (1999). They seem to be rather related to the type II burst. (It should additionally be noted that COSTEP has also detected a minor contribution of electrons in the range of $0.67-3.0 \mathrm{MeV}$.)

\subsubsection{Discussion: event on 19 May 1998}

The observations show that the filament was heated and erupted between 09:36.3-09:44 SRT. The CME, the EIT wave, and the driver of the shock wave associated with the type II burst started almost simultaneously and were generated due to the filament eruption. The following weak $\mathrm{X}$-ray flare (B7.9) was also initiated by the filament erup- tion and occured after the type II onset. It reached its maximum with a delay of $8 \mathrm{~min}$ with respect to the onset of the electron event. The very weak enhancement of the hard $\mathrm{X}$-ray flux and the soft $\mathrm{X}$-ray derivative indicates a possible nonthermal energy release in the low corona which might give some contribution to the electron flux observed by COSTEP/Wind 3-DP. But that occurred about $4 \mathrm{~min}$ after the electron event onset. There were no other variations either in the soft $\mathrm{X}$-ray derivative or in the hard X-ray emission between the type II onset and the onset of the appearance of the mildly relativistic electrons. Furthermore, no type III, IV or other radio bursts were observed at the frequencies above those of the type II burst.

All these facts allow us to suggest that the observed mildly relativistic electrons (inclusive the SA type III electrons) were produced by the coronal shock. There are no other indications of acceleration processes in the low corona at least before and during the onset of the electron event.

We suppose three stages:

1. a fast filament darkening and slow upward motion of the filament material seen in EIT at 08:54-09:25 SRT, probably due to the global destabilization of the magnetic field below and around the filament;

2. a sudden heating and eruption of the filament at about 09:37 SRT, accompanied by an arcade of soft X-ray and EUV loops forming beneath and along the filament (already seen at 09:47 SRT). The part of the filament is also heated and seen in X-ray and EUV emission. That produces a disturbance which was responsible for the CME and EIT wave formation. Later, at 09:44 SRT, it was transformed into the shock wave seen as a type II burst. If the EIT wave starts at 09:37 SRT then it has a velocity of $600 \mathrm{~km} \mathrm{~s}^{-1}$, which is in agreement with the CME speed of $650 \mathrm{~km} \mathrm{~s}^{-1}$ as estimated from LASCO images (height-time plot in Fig. 2, and Table 1). The filament speed is $457 \mathrm{~km} \mathrm{~s}^{-1}$. High energy electrons were not observed before the type II burst formation;

3. from its onset the type II burst is accompanied by SA type III bursts, which emanate from its lanes. Later, when the shock and the CME reached heights of 1.4$1.9 R_{\mathrm{S}}$ and the production of the SA type III bursts ceased, the mildly relativistic electrons were released.

\subsection{Event on 20 April 1998}

A strong electron event was associated with a M1.4 X-ray flare (start at 09:12, peak at 10:13 SRT) and a partial halo CME first detected at 09:59 SRT. In the radio domain the flare was accompanied by weak continuum emission starting in the range 150-400 MHz at 09:12 SRT (Fig. 3). From 09:28 SRT, the continuum began a slow drift from 400 to $100 \mathrm{MHz}$. A type II burst started at $55 \mathrm{MHz}$ at 09:48 SRT and disappeared at $4 \mathrm{MHz}$ at 10:42 SRT. Its source moved outwards with a velocity of $1150 \mathrm{~km} \mathrm{~s}^{-1}$ in the corona. We identified only one lane, hence it is not clear if 


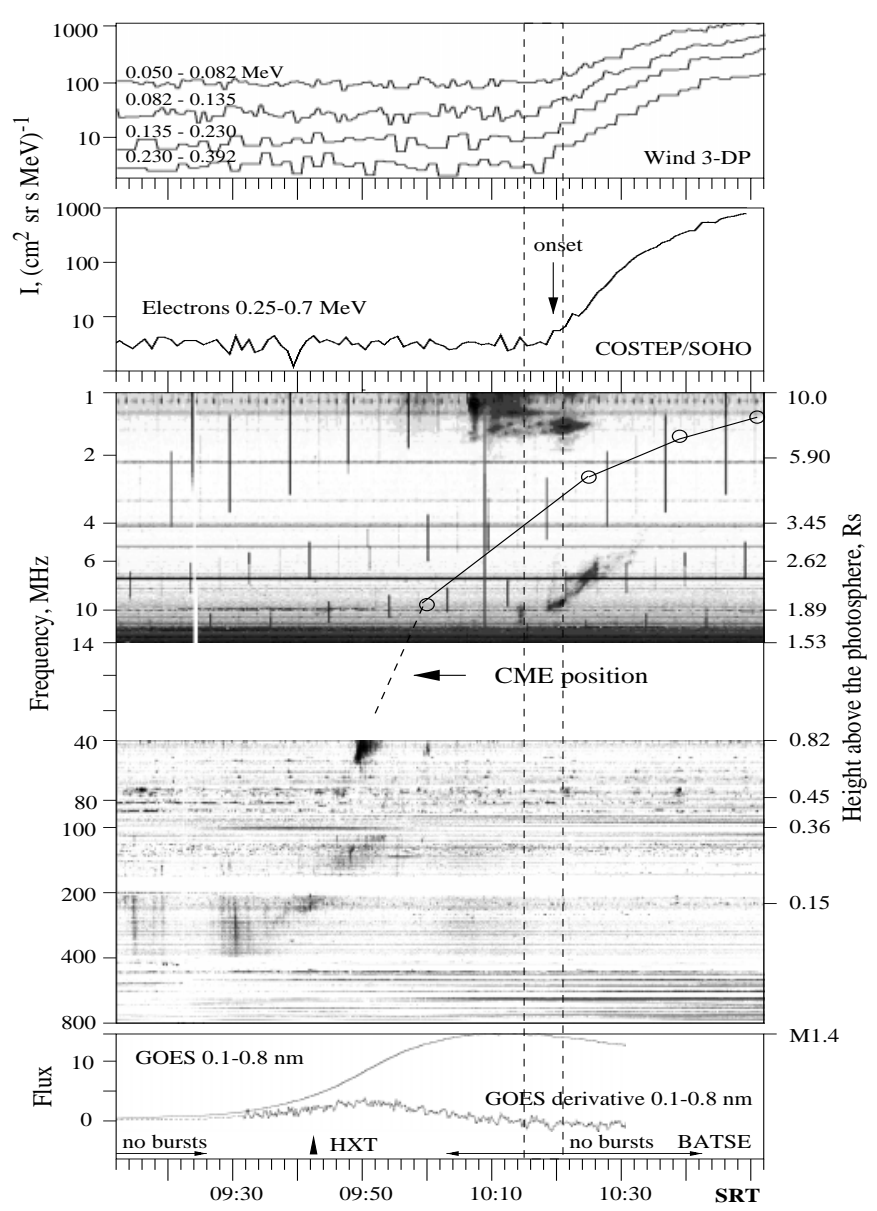

Fig. 3. Event on 20 April 1998. The electron event starts during the type II burst and the CME passage through the corona, but 30 min after the type II onset. The triangle at the bottom panel point to a weak HXR burst ( 2 counts, $33-53 \mathrm{keV}$ ). During the ejection of mildly relativistic electrons there are no HXR bursts.

the fundamental or harmonic mode was observed. The SA type III bursts occured from 09:52 to 10:26 SRT, visible below $3 \mathrm{MHz}$. Using radio imaging data of the $\mathrm{NRH}$, Maia et al. (2000) found that the drifting continuum appearing at 09:46 SRT was closely associated with the CME. They interpreted this continuum emission as type II-like emission that is somewhat misleading. The NRH cannot see the type II source at $164 \mathrm{MHz}$, because the type II started at $55 \mathrm{MHz}$. The close association between such a continuum and CMEs was also found by Aurass et al. (1999). Bastian et al. (2001) first discovered a radio CME on 20 April 1998 using the NRH observation. Its parameters are in agreement with the white light CME observed by LASCO. They suggested that the radio CME is due to synchrotron emission of relativistic electrons trapped in closed magnetic field structures. The relation between the radio CME and the type II source is unknown because of missing radio imaging data between 55 and $4 \mathrm{MHz}$. If we assume that the observed type II lane is the harmonic mode then the fundamental lane would be in good agreement with the CME height-time plot (Fig. 3).

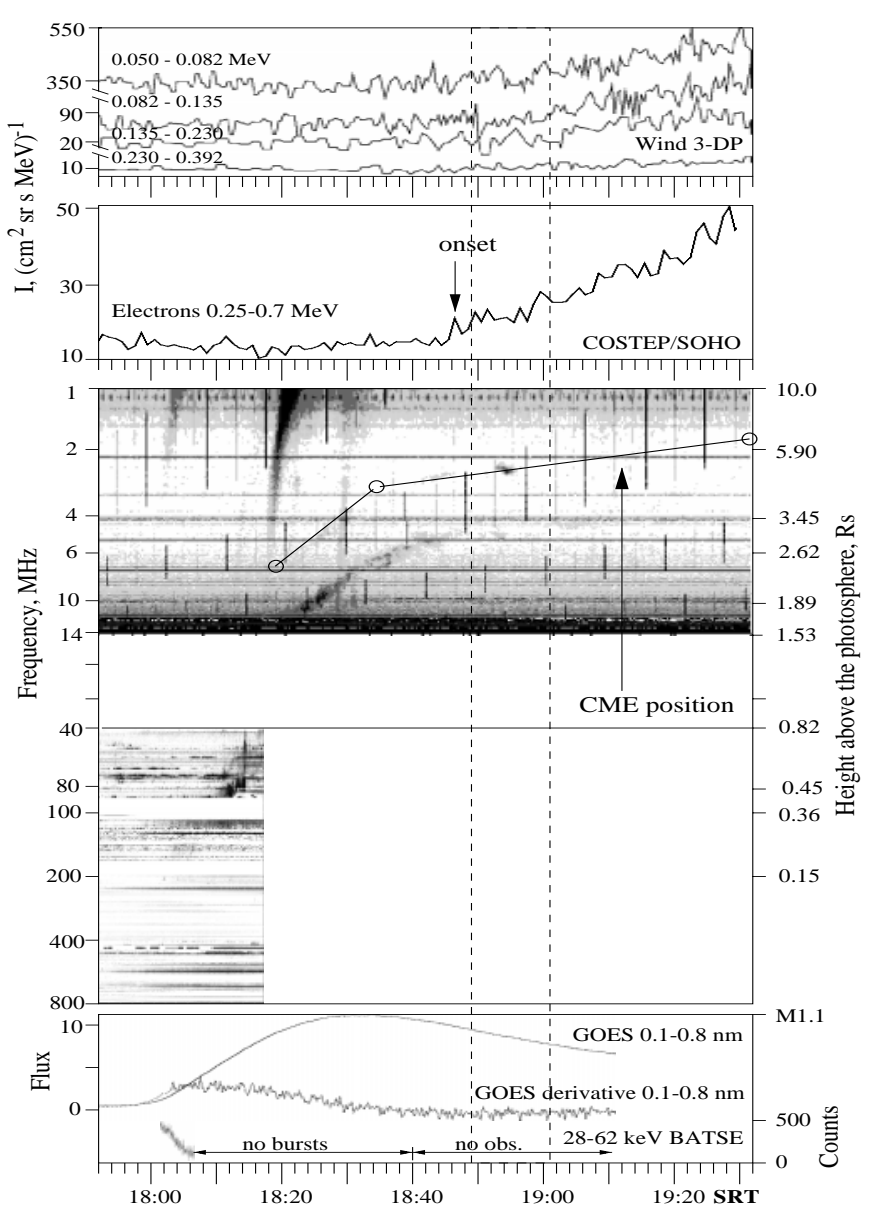

Fig. 4. Event on 16 June 1998. The mildly relativistic electrons $(0.25-0.7 \mathrm{MeV})$ starts $45 \mathrm{~min}$ after the type II onset, when the type II burst and the CME were at $4-5 R_{\mathrm{s}}$. Data gap after 18:17 SRT is due to the sunset. There are no other radio bursts during and at frequencies above those of type II (SGD-Solar Geophysical Data).

The electron event starts simultaneously in all channels at 10:19 $\pm 2 \mathrm{~min}$ (Fig. 3), when the CME and the type II burst reached a height of $2.5-4.0 R_{\mathrm{s}}$ above the photosphere. The time delay between the start of the type II and the electron event was $30 \mathrm{~min}$. It is important to note that the relativistic electrons were released without any radio emission from microwaves down to the metric domain. Only a radio continuum (09:57-10:21 SRT, maximum at 10:05 SRT, at 100-400 MHz) associated with the radio $\mathrm{CME}$ is seen shortly before the electron event onset (Bastian et al. 2001). The SXR flux, its derivative and the HXR observations show no enhancements or indications of acceleration in the low corona during the onset of the electron event (Fig. 3). Again, these results suggest that the SA type III electrons and the mildly relativistic electrons (0.25-0.7 MeV) were produced by the coronal shock.

\subsection{Event on 16 June 1998}

This event was associated with a M1.0 flare on the SW limb. Figure 4 shows the time profiles of electron 
intensities in the range $0.050-0.7 \mathrm{MeV}$, the CME heighttime plot superposed on dynamic radio spectrum, the soft $\mathrm{X}$-ray flux, its derivative and HXR BATSE flux registration.

In the metric domain the type II started around $170 \mathrm{MHz}$ at 18:02 SRT and drifted to $40 \mathrm{MHz}$ at 18:17 SRT (later data are not available). In the decametric domain both the fundamental and the harmonic mode of type II emission were observed. They started at about 18:22 SRT with the harmonic (fundamental) at $11.5 \mathrm{MHz}(5.6 \mathrm{MHz})$. From 18:22 SRT until about 18:52 SRT, these type II lanes drifted to $4 \mathrm{MHz}$ and $2 \mathrm{MHz}$ for the harmonic and fundamental lane, respectively. There are no other radio bursts recorded before and during as well as at frequencies above those of the type II burst. There is a large frequency (and therefore density) jump between the metric type II burst at $40 \mathrm{MHz}$ and the decametric type II at $12 \mathrm{MHz}$ at about 18:20 SRT. The low-frequency extrapolation of the metric type II frequency drift did not match the observed frequency drift of the type II burst in the range 14-6 MHz. This suggests that the metric and the decametric type II radio emission were generated by distinct shocks (see e.g. Reiner et al. 2000). The other possibility is that metric and decametric radio emission were produced by the same disturbance passing along different trajectories through regions of different plasma densities. In fact the metric type II bursts normally have a strong azimuthal speed component (Klassen et al. 1999). The mildly relativistic electrons were firstly released at 18:47 SRT (COSTEP) and at 18:55 SRT \pm 6 min (Wind 3-DP) during the type II burst and the CME, but after the SA type III bursts and the flare maximum.

The time delay between the start of the metric type II burst and the electron event was $45 \mathrm{~min}$. The discrepancy in the estimation of electron injection time derived from the data of both instruments is mainly due to the low electron intensity. The electrons were released when both the type II burst and the CME were at $4-5 R_{\mathrm{s}}$.

The possible contribution of activity in the low corona to the electron flux was minimal, because the SXR time derivative show no indications at an energy release and there are no reports about other radio bursts in this time interval.

\subsection{Event on 18 February 2000}

This event was associated with a small C1.1/SF flare near the SW limb. It started at 09:13 SRT and peaked at 09:19 SRT. The dynamic radio spectra (Fig. 5) shows a group of multilane type II burst between 170 and $40 \mathrm{MHz}$ in time interval of 09:10-09:42 SRT. The SA type III bursts occured at the frequencies below and during the type II emission. No other radio emission is observed from metric-to-centimetric wavelengths. The CME and the type II burst have a velocity of 940 and $580-1020 \mathrm{~km} \mathrm{~s}^{-1}$, respectively. Note that noise storm emission was present in the range of 400-100 MHz during the whole day. Using the

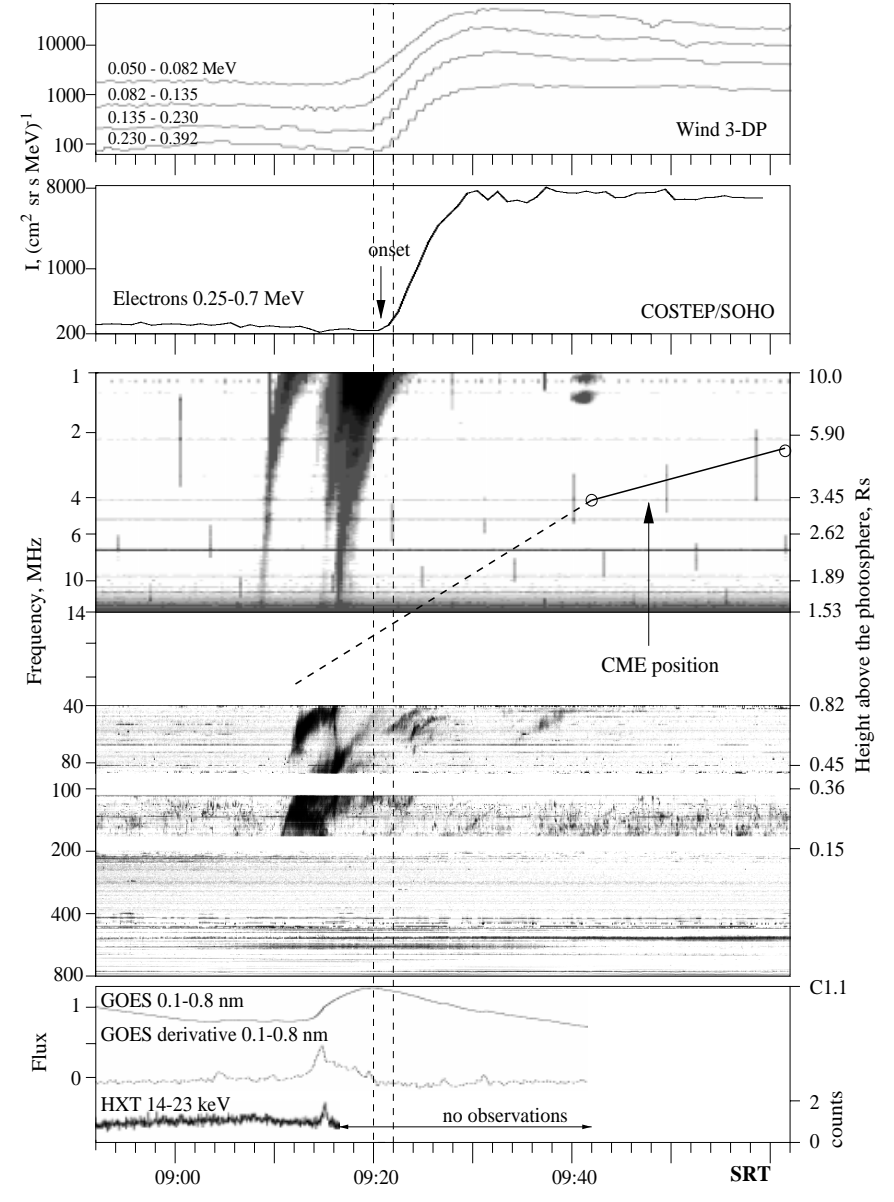

Fig. 5. Event on 18 February 2000. The electron event starts during the type II and the CME, but 11.5 min after the type II onset. There are no radio bursts at the frequencies above those of type II in the metric-to-centimetric range.

$\mathrm{NRH}$ observations at $164 \mathrm{MHz}$ we found that the type II bursts showed a source at an height of $0.40-0.54 R_{\mathrm{s}}$. This confirms the used twofold Newkirk model as a good approximation for this event. In Fig. 5 (top) the electron fluxes measured by the Wind 3-DP instrument do not show a clear velocity dispersion, due to the instrumental effect (Krucker et al. 2002). This effect is small for the highest energy channel. Therefore, the onset time of $0.392 \mathrm{MeV}$ electrons is used to determine the solar release time.

As for the 19 May 1998 event, the electron event started at the end of the SA bursts with a time delay of 11.5 min with respect to the type II burst onset, when the type II source and the CME reached the height of 1$1.5 R_{\mathrm{s}}$. About 5 min before the onset of the electron event a weak HXR burst was observed in the range $14-23 \mathrm{keV}$ by HXT/Yohkoh in agreement with the SXR derivative. During the electron event onset there are no peaks in the SXR derivative and no radio bursts at frequencies above those of the type II burst (note there are no HXR observations). Therefore, we suggest that the relativistic electrons in the range $0.2-0.7 \mathrm{MeV}$ were accelerated by the coronal shock. 
Table 2. Electron parameters.

\begin{tabular}{llc}
\hline \hline Event & $\begin{array}{l}\text { Peak flux, } \\
\text { at 27 keV }\end{array}$ & $\begin{array}{c}\text { Spectral index, } \\
\text { near 100 keV }\end{array}$ \\
\hline 20 April 1998 & 0.1 & -2.4 \\
19 May 1998 & 0.01 & -3.2 \\
16 June 1998 & 0.002 & -2.1 \\
18 February 2000 & 0.2 & -2.7 \\
\hline
\end{tabular}

Peak flux in erg $\left(\mathrm{cm}^{2} \mathrm{~s} \text { sterad } \mathrm{eV}\right)^{-1}$.

\section{Electron energy spectra}

The observed electron energy spectra of the selected solar events show a power-law shape distribution with spectral indices near $100 \mathrm{keV}$ between -3.2 and -2.1 (Table 2). The electron peak fluxes ranged between $0.1-$ $0.002 \mathrm{erg}\left(\mathrm{cm}^{2} \mathrm{~s} \text { sterad } \mathrm{eV}\right)^{-1}$, i.e. from small to large IP events.

The events show somewhat harder power-law spectra (spectral index $>-3.2$ ) than the mean spectral index $(-3.3)$ of all electron events observed by Wind 3-DP (Oakley et al. 2002). They found that the spectral index ranged between -4.5 and -2 , with a clear peak near -3.3 .

\section{Discussion}

Our study confirm the existence of two populations of energetic electrons: the low energetic beams associated with the type III radio emission and the high energy electrons released in the corona after the first population (Krucker et al. 1999; Roelof \& Haggerty 2001). Additionally we found that both populations can be accelerated by the same driver, i.e. by the coronal shock in association with CME, without influence of an impulsive flare phase. Of course the latter can also produce the whole spectrum of energetic electrons (e.g. Dulk et al. 1985).

The first population show distinct evidency that the coronal shocks produce energetic electrons in the range below $30 \mathrm{keV}$, because the HB and SA type III bursts escape from the type II backbones. These electrons are released just after the start of type II bursts, but they were not detected by the COSTEP and Wind 3-DP instruments. It is possible that due to the geometrical reasons the type III electrons were not ejected towards the Earth/spacecraft. The second population (range above $0.25 \mathrm{MeV}$ ) is released later, when the shock and the CME reached the height of $1-4 R_{\mathrm{S}}$, without any corresponding signatures in radio emission. Furthermore in all four events COSTEP have also detected electrons in energy channel of $0.67-3.0 \mathrm{MeV}$.

What is the reason that the mildly relativistic electrons were not accelerated together with the onset of type II bursts but rather more than 11 min later?

We see two main scenarios to explain this result: In the first scenario, both populations are accelerated simultaneously, but the mildly relativistic electrons are trapped in the corona and are ejected later when the shock and the CME reach heights of $h \sim 1-4 R_{\mathrm{s}}$ where the magnetic field structure is predominantly open or is opened up during the event. For example, the event on 18 February 2000 showed an indication of nonthermal energy release in the low corona about 5 min before the high energy electrons were released (see Fig. 5). This activity might contribute to the observed relativistic electrons. In this case the electrons should be trapped at least for $5 \mathrm{~min}$ in the corona. Later, during the type II occurrence they are ejected in the IP medium without signatures in the low corona, particularly in the radio domain and in the SXR derivative. The observation of the radio CME on 20 April 1998 (Bastian et al. 2001) occurring due to synchrotron radiation of relativistic electrons trapped in closed magnetic field structures at the heights of some solar radii supports this scenario.

However there are reasonable objections against this scenario. The trapped electrons should give rise to continuum (e.g. type IV) radio emission. This is observed only in one case (20 April 1998). The observed shocks produce energetic beams of electrons (SA type III bursts) just after their onset. They propagate through the corona into the IP medium. That means there must already be open magnetic field lines, along which the electrons of both populations could escape. But we detect only the second population with a time delay of more than 11 min. Why?

As a result of these discrepancies another scenario seems to be more likely: both populations are accelerated with some time delay. The mildly relativistic electrons are released at the end or after the SA type III radio bursts, which indicates that one acceleration regime is switched off and the other one is switched on. Perhaps it is due to the different regimes of coronal shock. At heights of $\sim 1-4 R_{\mathrm{S}}$ the shocks more likely travel parallel to the magnetic field, whereas at low heights the shocks are more perpendicular. Krucker et al. (1999) found at least two events which clearly show that electrons of both classes can occur during the same solar event, but the electrons with higher energies are released later.

Alternative candidates that can indicate the relativistic electron acceleration processes in the low corona such as HXR bursts, radio bursts (type III, IV etc.) were absent or did not occur during the onset of relativistic electrons. Indeed: (a) there are no other radio bursts at the frequencies above those of type II, (b) in two of our 4 events there are no HXR bursts, whereas no HXR observations were available for the other ones, (c) the SXR derivatives show no indication of the energy release in the low corona in all events. Therefore it is plausible to suggest that the best canditate for observed relativistic electron events is the coronal shock in association with CME.

\section{Conclusions}

The investigation of four solar energetic electron events and the associated activity in terms of type II bursts, CMEs, EIT waves etc. has revealed the existence of two populations of energetic electrons released during the passage of the shock wave and the CME through the corona. 
The possible contributions of acceleration processes in the low corona to the observed relativistic electron events were negligible or minimal. The first electron population is associated with SA type III radio bursts and are released just after the onset of type II bursts. For the second population - the mildly relativistic electrons - we found that:

1. all of the $0.25-0.7 \mathrm{MeV}$ electron events were released during or after, but never simultaneously with the onset of type II bursts and CMEs. The time delay between type II burst onset and electron event release ranged from 11.5 to $45 \mathrm{~min}$;

2. the electrons were released rather at the end of SA type III bursts or somewhat later;

3. the electrons were released when the associated type II burst and the CME reached a certain height above the photosphere $\left(h \sim 1-4 R_{\mathrm{S}}\right)$.

We suggest that both populations of electrons are not only ejected (released), but also accelerated by the coronal shock with some time delay relative to each other.

Acknowledgements. We would like to thank H. Aurass and G. Trottet for helpful discussions and comments. We are thankful to J. Khan for help with the processing and access of Yohkoh-SXT-HXT data at the Mullard Space Science Laboratory (UK) and to B. J. Thompson for providing $S O H O / E I T$ images (SOHO/EIT Consortium). The $\mathrm{H}_{\alpha}$ images were obtained via internet from KiepenheuerInstitut für Sonnenphysik (Freiburg) and from Learmonth Solar Observatory (Learmonth). GOES and BATSE/CGRO data were provided by the Solar Data Analysis Center at NASA/GSFC. The work of A. Klassen is supported by the Deutsche Forschungsgemeinschaft (grant DFG MA1376/14-1).

\section{References}

Aurass, H., Vourlidas, A., Andrews, M. D., et al. 1999, ApJ, 511,451

Bastian, T. S., Pick, M., Kerdraon, A., et al. 2001, ApJ, 558, L65

Bothmer, V., Posner, A., Kunow, H., et al. 1997, ESA SP-415, 207

Bougeret, J.-L., Kaiser, M. L., Kellogg, P., et al. 1995, Space Sci. Rev., 71, 231

Bougeret, J.-L., Zarka, P., Caroubalos, C., et al. 1998, Geophys. Res. Lett., 25, 2513

Bruzek, A. 1958, Z. Astrophys., 44, 183
Brueckner, G. E., Howard, R. A., Koomen, M. J., et al. 1995, Sol. Phys., 162, 357

Cairns, I. H., \& Robinson, R. D. 1987, Sol. Phys., 111, 365

Cane, H. V., Stone, R. G., Fainberg, J., et al. 1981, Geophys. Res. Lett., 8, 1285

Dulk, G. A., McLean, D. J., \& Nelson, G. J. 1985, in Solar Radiophysics, ed. D. J. McLean, N. R. \& Labrum (Cambridge Univ. Press, Cambridge), 53

Dulk, G. A., Leblanc, Y., Bastian, T. S., \& Bougeret, J.-L. 2000, JGR, 105, 27343

Gopalswamy, N., Lara, A., Kaiser, M. L., \& Bougeret, J.-L. 2002, JGR, in press

Kahler, S. W. 1982, ApJ, 261, 710

Klassen, A., Aurass, H., Klein, K.-L., et al. 1999, A\&A, 343, 287

Klein, K.-L., Anderson, K., Pick, M., et al. 1983, Sol. Phys., 84, 295

Klein, K.-L. 1995, in Coronal Magnetic Energy Releases, ed. A. O. Benz, \& A. Krüger (Lecture Notes in Physics, Springer), 55

Krucker, S., Larson, D. E., Lin, R., \& Thompson, B. J. 1999, ApJ, 519, 864

Krucker, S., Lin, R. P., Bale, S. D., \& Larson, D. E. 2002, ApJ, in press

Laitinen, T., Klein, K.-L., Kocharov, L., et al. 2000, A\&A, 360, 729

Lin, R. P., Anderson, K. A., Ashford, S., et al. 1995, Space Sci. Rev., 71, 125

Mann, G., Jansen, F., MacDowall, R. J., et al. 1999, A\&A, 348,614

Mann, G., Aurass, H., Voigt, W., \& Paschke, J. 1992, ESAJournal SP-348,129

Maia, D., Pick, M., Vourlidas, A., \& Howard, R. 2000, ApJ, 528, L49

Martin, S. F., \& Ramsey, H. E. 1972, in Solar activity observations and predictions, ed. P. S. Mcintosh, \& M. Dryer (Mass. Inst. of Technology), 301

Müller-Mellin, R., Kunow, H., Fleissner, V., et al. 1995, Sol. Phys., 162, 483

Newkirk, G. A. 1961, ApJ, 133, 983

Oakley, P. H., Krucker, S., \& Lin, R. P. 2002, ApJ, in press

Reames, D. V. 1999, Space Sci. Rev., 90, 413

Reiner, M. J., Kaiser, M. L., Plunkett, S. P., et al. 2000, ApJ, 529, L53

Roberts, J. A. 1959, Austr. J. Phys., 12, 327

Roelof, E. C., \& Haggerty, D. K. 2001, in abstracts AGU Spring Meeting, Boston

Smith, S. F., \& Ramsey, H. E. 1964, Z. Astrophys., 60, 1

Stewart, R. T. 1976, Sol. Phys., 50, 437

Vršnak, B. 2001, JGR, 106, 25291 